\title{
Diagnosis and treatment of multidrug-resistant tuberculosis
}

\section{Jong Geol Jang, Jin Hong Chung}

Department of Internal Medicine, Yeungnam University College of Medicine, Daegu, Korea

Received: July 14, 2020

Revised: August 21, 2020

Accepted: August 25, 2020

Corresponding author:

Jin Hong Chung

Department of Internal Medicine,

Yeungnam University College of

Medicine, 170 Hyeonchung-ro,

Nam-gu, Daegu 42415, Korea

Tel: +82-53-640-6682

Fax: +82-53-620-3849

E-mail:jhchn@med.yu.ac.kr
Tuberculosis (TB) is still a major health problem worldwide. Especially, multidrug-resistant TB (MDR-TB), which is defined as TB that shows resistance to both isoniazid and rifampicin, is a barrier in the treatment of TB. Globally, approximately $3.4 \%$ of new TB patients and 20\% of the patients with a history of previous treatment for TB were diagnosed with MDR-TB. The treatment of MDR-TB requires medications for a long duration (up to 20-24 months) with less effective and toxic second-line drugs and has unfavorable outcomes. However, treatment outcomes are expected to improve due to the introduction of a new agent (bedaquiline), repurposed drugs (linezolid, clofazimine, and cycloserine), and technological advancement in rapid drug sensitivity testing. The World Health Organization (WHO) released a rapid communication in 2018, followed by consolidated guidelines for the treatment of MDR-TB in 2019 based on clinical trials and an individual patient data meta-analysis. In these guidelines, the WHO suggested reclassification of second-line anti-TB drugs and recommended oral treatment regimens that included the new and repurposed agents. The aims of this article are to review the treatment strategies of MDR-TB based on the 2019 WHO guidelines regarding the management of MDR-TB and the diagnostic techniques for detecting resistance, including phenotypic and molecular drug sensitivity tests.

Keywords: Diagnosis; Multidrug-resistant tuberculosis; Treatment

\section{Introduction}

Tuberculosis (TB) is the tenth leading cause of death worldwide and a major global health problem [1]. The World Health Organization (WHO) reported that 10.4 million patients developed $\mathrm{TB}$ and 1.6 million patients died from TB worldwide in 2017 [2]. Drug resistance is one of the major threats to the treatment of TB. The WHO has defined multidrug-resistant TB (MDR-TB) as TB that shows resistance to isoniazid as well as rifampicin, the most effective anti-TB drugs [3]. In 2018, a total of 186,772 cases were diagnosed with MDR-TB and rifampicin-resistant TB, and 156,071 patients began treatment worldwide [4]. Approximately $3.4 \%$ of the new TB patients and $20 \%$ of the patients with a history of previous treatment for $\mathrm{TB}$ were diagnosed with MDR-TB worldwide
[4]. Treatment of MDR-TB lasts for a long duration of approximately 2 years and consists of a combination of multiple second-line drugs, which are more expensive, less effective, and more toxic than the first-line drugs. Therefore, treatment outcomes for MDR-TB are poor, with a success rate of approximately $54 \%$ [2]. WHO published new guidelines for MDR-TB treatment in 2019. This article reviews the treatment of MDR-TB according to the most recent updated WHO guidelines and diagnosis of MDR-TB [2].

\section{Definitions of tuberculosis drug resistance}

- Mono-resistant TB is defined as TB caused by an isolate that shows resistance to a single first-line anti-TB drug (isoniazid, rifampicin, ethambutol, or pyrazinamide) [5].

Copyright (C) 2020 Yeungnam University College of Medicine

This is an Open Access article distributed under the terms of the Creative Commons Attribution Non-Commercial License (http://creativecommons.org/licenses/by-nc/4.0/) which permits unrestricted non-commercial use, distribution, and reproduction in any medium, provided the original work is properly cited. 
- Isoniazid-resistant TB is defined as TB caused by an isolate that shows resistance to isoniazid, but is susceptible to rifampicin.

- Rifampicin-resistant TB is defined as TB caused by an isolate that shows resistance to rifampicin, but is susceptible to isoniazid.

- Poly-resistant TB is defined as TB caused by an isolate that is resistant to more than one anti-TB drug, but not resistant to both isoniazid and rifampicin simultaneously.

- MDR-TB is defined as TB caused by an isolate that shows resistance to at least isoniazid and rifampicin.

- Pre-extensively drug-resistant TB is defined as TB caused by an isolate that shows resistance to isoniazid, rifampicin, and either fluoroquinolones or injectable agents (amikacin, kanamycin, or capreomycin), but not both.

- Extensively drug-resistant TB is a rare type of MDR-TB that is resistant to isoniazid and rifampicin as well as to any fluoroquinolone and at least one out of the three injectable agents (amikacin, kanamycin, or capreomycin). Approximately $9 \%$ of the MDR-TB patients have extensively drug-resistant TB.

\section{Mechanism of drug resistance}

Drug resistance to Mycobacterium tuberculosis (MTB) results from spontaneous and random chromosomal mutations that result in reduced susceptibility to specific agents [6]. The mechanism leading to the development of drug resistance includes activation of the efflux pump at the surface of the bacteria, drug target alteration, production of drug inactivating enzymes, and disruption of drug activation [7]. The incidence of MDR-TB is low, as the rate of mutation is $10^{-5}$ for isoniazid and $10^{-7}$ for rifampicin [8]. Drug resistance can occur in two ways (primary or secondary resistance). Primary resistance develops when patients are exposed to and infected with an already drug-resistant strain. Secondary resistance or acquired resistance develops due to poor adherence to medication, drug malabsorption, and inadequate regimen among patients taking TB medication. Although most cases of MDR-TB arise from acquired resistance, a previous study reported that most of the incidences of MDR-TB resulted from transmission rather than acquisition of resistance during treatment in most high-burden settings [9].

\section{Diagnosis of multidrug-resistant tuberculosis}

Successful diagnosis and treatment of MDR-TB are based on a rapid and precise drug sensitivity test (DST), which provides evidence for selecting an effective drug [4]. DST is divided into phenotypic tests that observe growth or metabolic inhibition in an-
ti-TB drug-free and drug-containing media and molecular tests that detect genes related to drug resistance [7]. Conventional phenotypic DST is a solid culture-based method that uses egg-based or agar-based media. There are three different methods, namely: the proportion method, the resistance ratio method, and the absolute concentration method $[10,11]$. The proportion method is the most commonly used method. It is the reference method for phenotypic testing, which provides a measure of the susceptibility of the bacteria to a drug $[11,12]$. The absolute concentration method is also commonly used due to its technical convenience [7]. These methods are sensitive, have good clinical correlation, and enable the determination of minimal inhibitory concentration. However, it takes a relatively long time as long as 2 to 3 months to confirm the DST results due to the long turnaround time for MTB culture [13]. Liquid culture and DST have a higher rate of MTB isolation and require less time for detection than solid culture and DST. However, it is more expensive and carries a risk of increased bacterial contamination and cross infection by nontuberculous mycobacterial isolation [14]. In order to shorten the turnaround time for mycobacterial culture and DST, a variable rapid culture technique has been developed that usually utilizes liquid media (BACTEC 460, Becton Dickinson, Sparks, MD, USA; Mycobacteria Growth Indicator Tube [MGIT], Becton Dickinson; Septi-Check, Becton Dickinson; Myco-ESP Culture System II, Trek Diagnostic Systems, Westlake, OH, USA; BacT/ALERT MB susceptibility kit, bioMérieux Inc., Durham, NC, USA). This technique can provide DST results within a month [15]. Among the liquid-based culture systems, the most commonly used systems are BACTEC 460 that detects carbon dioxide production and MGIT that detects oxygen consumption [14].

Molecular DSTs have been developed to offer an advantage over conventional phenotypic tests that are more time-consuming. These tests can be used to diagnose TB through amplification of nucleic acids. They detect drug resistance by identifying genetic mutations in specific genes. These genotypic tests are more rapid and accurate than the phenotypic DSTs [16]. Molecular DSTs are divided broadly into two types; probe-based assays and sequence-based assays.

The probe-based DSTs include line probe assays (LPA) and GeneXpert (Cepheid Inc., Sunnyvale, CA, USA). In 2008, WHO approved the use of commercial LPAs (the INNO-LiPA Rif.TB assay [Innogenetics, Ghent, Belgium] and the GenoType MTBDRplus version 1 [MTBDRplus; Hain Lifescience GmbH, Nehren, Germany]) for detecting MTB and drug resistance [17]. In 2015, WHO performed a systemic review of the accuracy of commercial LPAs (MTBDR plus version 1, version 2, and Nipro NTM+MTBDR [NIPRO Corp., Osaka, Japan]) for detecting MTB 
and resistance to isoniazid and rifampicin, and later in 2016, WHO recommended the use of LPAs in patients with culture-positive (direct testing) or a sputum smear-positive specimens (indirect testing) $[18,19]$. The MTBDRplus is a semi-automated genotypic method that consists of three steps, namely DNA extraction, multiplex polymerase chain reaction (PCR) amplification, and reverse hybridization. This method can detect mutations in the rpoB gene for rifampicin resistance and in the $k a t \mathrm{G}$ gene and the inh $\mathrm{A}$ promoter region for isoniazid resistance [20,21]. Although MTBDRplus has shown high accuracy for rifampicin resistance (98.7\%), its accuracy for isoniazid is variable and has relatively low sensitivity (84.3\%) [22]. Recently, the WHO recommended the GenoType MTBDRsl (Hain Lifescience $\mathrm{GmbH}$ ) that was developed to detect resistance to ethambutol (mutation in $e m b \mathrm{~B}$ ), fluoroquinolones (mutations in $g y r \mathrm{~A}$ and $g y r \mathrm{~B}$ ), and injectable agents (mutation in rrs, leading to resistance to kanamycin, amikacin, and capreomycin) [23].

In 2020, the updated WHO guidelines recommended the use of molecular assays (Xpert MTB/RIF and Xpert MTB/RIF [Xpert Ultra]; GeneXpert) as the initial test for the diagnosis of pulmonary and extrapulmonary TB and rifampicin resistance in adults and children $[19,24]$. The Xpert MTB/RIF is a fully automated real-time PCR based molecular assay for detecting MTB and resistance to rifampicin [25], which provides results within 2 hours. In a large clinical trial, the Xpert MTB/RIF showed an MTB detection accuracy of $98.2 \%$ in smear-positive and culture-positive patients, but the accuracy was $72.5 \%$ in smear-negative and culture-positive patients. The specificity of the Xpert MTB/RIF was 99.2\%. In the same study, the Xpert MTB/RIF showed 97.6\% sensitivity for detecting rifampicin resistance [22]. The WHO also recommends Xpert MTB/RIF for the diagnosis of extrapulmonary TB (e.g., tuberculous lymphadenitis and tuberculous meningitis) based on a systematic review [26]. The Xpert Ultra was developed to improve the sensitivity of TB diagnosis (especially in smear-negative, human immunodeficiency virus [HIV]-infected patients and in case of extrapulmonary TB such as tuberculous meningitis and tuberculous lymphadenitis) and rifampicin resistance identification. For TB detection, the sensitivity of Xpert Ultra was higher than that of Xpert in smear-negative patients and in patients with HIV, but the specificity was lower than that of Xpert in all patients [27]. A recent study reported that Xpert Ultra was not superior to Xpert in diagnosing tuberculous meningitis [26]. Further evaluation of the diagnostic accuracy of Xpert Ultra is required. To date, there have been no fully automated molecular assays that can detect resistance to second-line agents. In Korea, rapid DST using LPA and Xpert can be used.

Probe-based DSTs are not able to detect resistance profiles when mutations occur outside the target genetic region [28]. Next-generation sequencing (NGS) is a technique that can compensate for this weakness. NGS provides rapid and detailed sequence information of a part of the genome (targeted NGS) or the whole genome (whole genome sequencing). It can identify genotypes that predict drug-resistant phenotypes. It can also provide genetic information that can detect transmission in potential outbreak situation [29]. This technique can provide drug susceptibility profiles not only for the first-line drugs but also for many second-line drugs [30]. Whole genome sequencing was well correlated with phenotypic DST as well as with culture conversion rate and treatment outcome [31]. However, NGS has several disadvantages, such as poor sensitivity while using sputum rather than culture isolate as a specimen and the need for specialized staff [32].

\section{Treatment of multidrug-resistant tuberculosis}

The goal of treatment for MDR-TB is to cure the individual patient and to avoid the transmission of MDR-TB to other people. The $\mathrm{WHO}$ developed guidelines for the programmatic management of drug-resistant TB in 2006 and updated these guidelines in 2011. These updated guidelines recommend the use of rapid diagnosis of rifampicin resistance and a combination of four effective drugs, including pyrazinamide, an injectable agent, and a later generation fluoroquinolone for the treatment of patients with MDR-TB [33]. In the updated guidelines of 2016, the WHO suggested MDR-TB regimens with at least five effective $\mathrm{TB}$ drugs, including pyrazinamide and four second-line TB drugs [5]. Drugs to be included in the regimen are fluoroquinolone, an injectable agent, ethionamide or prothionamide, pyrazinamide, and either cycloserine or $\mathrm{pa}$ ra-aminosalicylic acid (Table 1). Rapid DST for isoniazid and rifampicin or rifampicin alone is recommended. The WHO released a rapid communication in 2018 [34] and updated the consolidated guidelines in 2019 [2]. These guidelines include a new drug classification, guidelines for building regimens, enhanced monitoring strategies, and a feasible implementation plan based on clinical trials and individual patient data meta-analysis (IPD-MA) $[2,35,36]$. A recent IPD-MA including 12,030 patients from 25 countries involved analysis of anti-MDR-TB drugs associated with favorable outcomes. Treatment success was positively associated with the use of linezolid, levofloxacin, carbapenems, moxifloxacin, bedaquiline, and clofazimine. Reduced mortality was significantly associated with the use of linezolid, levofloxacin, moxifloxacin, and bedaquiline.

Streptomycin and amikacin provided modest benefits when compared with regimens without injectable agents [35]. Accord- 
Table 1. Classification of medication for multidrug-resistant tuberculosis in 2016

\begin{tabular}{|c|c|c|}
\hline Group & Classification & Medicine \\
\hline \multirow[t]{3}{*}{$A$} & Fluoroquinolones & Levofloxacin \\
\hline & & Moxifloxacin \\
\hline & & Gatifloxacin \\
\hline \multirow[t]{4}{*}{ B } & Second-line injectable agents & Amikacin \\
\hline & & Capreomycin \\
\hline & & Kanamycin \\
\hline & & Streptomycin \\
\hline \multirow[t]{4}{*}{ C } & Other core second-line agents & Ethionamide or prothionamide \\
\hline & & Cycloserine or terizidone \\
\hline & & Linezolid \\
\hline & & Clofazimine \\
\hline D & Add-on agents & \\
\hline \multirow[t]{3}{*}{ D1 } & & Pyrazinamide \\
\hline & & Ethabutol \\
\hline & & High-dose isoniazid \\
\hline \multirow[t]{2}{*}{ D2 } & & Bedaquiline \\
\hline & & Delamanid \\
\hline \multirow[t]{4}{*}{ D3 } & & Para-aminosalicylic acid \\
\hline & & Imipenem/cilastatin \\
\hline & & Meropenem \\
\hline & & Amoxicillin/clavulanate \\
\hline
\end{tabular}

Modified from World Health Organization treatment guidelines for drugresistant tuberculosis [5].

ing to the results of this IPD-MA, the updated guidelines have developed a new drug classification that divided drugs for MDR-TB into three groups $(\mathrm{A}, \mathrm{B}$, and $\mathrm{C})$ after prioritizing their effectiveness and toxicities (Table 2). Oral regimens are preferred for almost all patients. Fluoroquinolones (levofloxacin or moxifloxacin), bedaquiline, and linezolid are strongly recommended for a longer MDR-TB regimen. These three drugs should be included in the initial therapy unless there is an evidence of drug resistance or a risk of toxicity. In IPD-MA, when compared with injectable-free regimen, regimen including streptomycin or amikacin was associated with increased treatment success, while regimen including kanamycin or capreomycin showed poorer outcomes. Kanamycin treatment was associated with lower treatment success, and capreomycin was associated with lower success and higher mortality [35]. Injectable agents have critical toxicities (including hearing loss and nephrotoxicity) and poor adherence to drug. Considering the benefits and harms of injectable agents, they are not recommended in the initial MDR-TB regimen and have been downgraded to group C [35]. WHO recommended that amikacin and streptomycin be considered only when the patient's isolate is susceptible to these drugs and high-quality monitoring of hearing loss is possible. However, the poorer outcomes of injectable agents could
Table 2. Classification of medication for multidrug-resistant tuberculosis in 2019

\begin{tabular}{|c|c|c|}
\hline Group & Medicine & Step \\
\hline$A$ & $\begin{array}{l}\text { Levofloxacin or moxifloxacin } \\
\text { Bedaquiline } \\
\text { Linezolid }\end{array}$ & $\begin{array}{l}\text { Include all three medicines } \\
\text { (unless they cannot be used) }\end{array}$ \\
\hline B & $\begin{array}{l}\text { Clofazimine } \\
\text { Cycloserine or terizidone }\end{array}$ & $\begin{array}{l}\text { Add one or both medicines } \\
\text { (unless they cannot be used) }\end{array}$ \\
\hline C & $\begin{array}{l}\text { Ethambutol } \\
\text { Delamanid } \\
\text { Pyrazinamide } \\
\text { Imipenem-cilastatin or meropenem } \\
\text { Amikacin or streptomycin } \\
\text { Ethionamide or prothionamide } \\
\text { Para-aminosalicylic acid }\end{array}$ & $\begin{array}{l}\text { Add to complete a four- to } \\
\text { five drug regimen when } \\
\text { medicines from groups A } \\
\text { and B cannot be used }\end{array}$ \\
\hline
\end{tabular}

Modified from World Health Organization consolidated guidelines on drug-resistant tuberculosis treatment [2].

be attributed to several confounding factors, such as drug changes during the treatment, misclassification of treatment outcomes, and their selective use in severe clinical cases [35]. Although Korean guidelines also excluded kanamycin in classification of MDR-TB drug, they recommended that kanamycin can be used as a substitute for amikacin until additional data are available [37].

\section{Classification of drugs}

In 2018, the WHO rapid communication classified the drugs for the longer MDR-TB regimen into three groups (Table 2) [34]. Agents in group A include fluoroquinolones, bedaquiline, and linezolid, which are highly effective and strongly recommended in the MDR-TB regimen unless contraindicated. Clofazimine and either cycloserine or terizidone are included in group $\mathrm{B}$. These drugs are conditionally recommended as the second choice. Group C drugs can be used when an adequate regimen cannot be formulated with agents from group A or group B. Agents in group C are ranked by the balance of benefits to toxicities. It includes all other drugs except high-dose isoniazid, amoxicillin-clavulanate, kanamycin, and capreomycin.

Fluoroquinolones are effective against growing as well as non-growing tuberculous bacilli and are well tolerated over the long treatment period. Fluoroquinolones inhibit DNA transcription and bacterial replication of MTB by interfering with DNA gyrase, which is a tetramer composed of two $\alpha$ and two $\beta$ subunits encoded by gyrA and gyrB genes [38]. Fluoroquinolone resistance in MTB is usually caused by mutations in the gyrA gene [39]. Fluoroquinolones have become a mainstay of regimens used to treat MDR-TB, as their mechanism of action is distinct from both isoniazid and rifampicin [40]. 
Levofloxacin and moxifloxacin are the two most frequently recommended agents, and the WHO has recommended the use of these drugs for the treatment of MDR-TB. The optimal dose of levofloxacin is $750 \mathrm{mg}$ once daily and that of moxifloxacin is 400 mg once daily. The study from South Korea reported that levofloxacin and moxifloxacin have similar effectiveness and side effects [41]. Adverse effects of fluoroquinolones include gastrointestinal trouble, problems related to the central nervous system, and QT interval prolongation. However permanent discontinuation of fluoroquinolones due to side effects was uncommon [42].

Linezolid is an oxazolidinone antibiotic that inhibits bacterial protein synthesis by preventing the fusion of $30 \mathrm{~S}$ and $50 \mathrm{~S}$ ribosomal subunits [43]. Linezolid was categorized as a "group 5" drug in the 2011 WHO guidelines for drug-resistant TB. Agents in group 5 were not recommended for use as core drugs, as there was insufficient evidence regarding their efficacy and safety [33]. However, the 2016 WHO update reclassified linezolid into group C, which includes other core second-line agents [5]. In 2018, in the rapid communication released by the $\mathrm{WHO}$ regarding treatment of MDR-TB, linezolid was further elevated to group A. The effectiveness of linezolid in the treatment of drug-resistant $\mathrm{TB}$ has been confirmed in clinical trial and meta-analysis $[35,43]$. The optimal duration of linezolid use has not been established, but its long-term administration (at least 6 months) was associated with treatment success [34]. Concerns have been raised about safety and toxicity of linezolid. Critical adverse effects of linezolid include peripheral neuropathy, myelosuppression with consequent anemia and thrombocytopenia, and optic neuropathy leading to disability and blindness [44]. In a recent IPD-MA, the incidence of permanent discontinuation due to adverse effects of linezolid was 16.3\% [44]. The optimal dose of linezolid is unclear. A variety of dosing strategies have been used for drug-resistant TB, which range from 300 to $1,200 \mathrm{mg}$ daily, with once-daily or twice-daily administration $[45,46]$. The $600-\mathrm{mg}$ daily dose was reported to be safer than the 1,200-mg dose without lowering its effectiveness [46]. The WHO also recommends a daily dose of $600 \mathrm{mg}$. Although some studies report that a daily dose of $300 \mathrm{mg}$ is effective and reduces toxicities [45], it is associated with a risk for development of drug resistance. Moreover, there is no sufficient evidence for initiating treatment with a 300-mg daily dose.

Bedaquiline is a diarylquinoline compound that specifically inhibits the adenosine triphosphate synthase by blocking the flow of mycobacterial proton pump [47]. Bedaquiline has a concentration-dependent bactericidal effect by causing cell death in both replicating and non-replicating mycobacteria [48]. The standard regimens including bedaquiline showed a reduction in time to culture conversion and a higher cure rate at 120 weeks when compared with a pla- cebo [49,50]. Common adverse events include QT prolongation, nausea/vomiting, and arthralgia/myalgia. Severe adverse events were reported in $2.8 \%$ of the patients [44]. Bedaquiline is well absorbed, and its absorption increases with food. According to the clinical data for safety, tolerability, and efficacy, the U.S. Food and Drug Administration approved the dose of $400 \mathrm{mg}$ daily for 14 days followed by $200 \mathrm{mg}$ three times weekly for 22 weeks [51].

Delamanid is a new anti-TB agent derived from the nitro-dihydro-imidazooxazole class of compounds that inhibits mycolic acid synthesis of bacterial cell wall. It has shown potent in vitro and in vivo activity against both drug-susceptible and drug-resistant strains of MTB in early clinical development $[52,53]$. Due to the lack of data in the 2018 IPD-MA, delamanid was classified in group $\mathrm{C}$, and $\mathrm{WHO}$ recommended conditionally that delamanid may be included in the treatment of patients with MDR-TB aged 3 years or more on the longer regimen $[2,35]$. However, several studies reported that delamanid-containing regimen was as effective and safe as bedaquiline [54-56]. Thus, Korean guidelines classified delamanid in group $\mathrm{C} 2$, and recommend that delamanid can be used as a substitute for bedaquiline (Table 3) [37].

\section{Building of regimen}

This review will focus on building of longer MDR-TB regimens according to the $\mathrm{WHO}$ guidelines [2], since the shorter MDR-TB regimens are fixed. The regimens should include all three drugs from group $\mathrm{A}$ and at least one drug from group $\mathrm{B}$. Thus, the regimens should include at least four effective drugs (ideally five

Table 3. Classification of medication for multidrug-resistant tuberculosis in updated Korean guidelines

\begin{tabular}{|c|c|}
\hline Group & Medicine \\
\hline \multirow[t]{3}{*}{ A } & Levofloxacin or moxifloxacin \\
\hline & Bedaquiline \\
\hline & Linezolid \\
\hline \multirow[t]{2}{*}{ B } & Clofazimine \\
\hline & Cycloserine \\
\hline \multicolumn{2}{|l|}{$\mathrm{C}$} \\
\hline \multirow[t]{6}{*}{$C 1^{\mathrm{a})}$} & Amikacin (streptomycin) ${ }^{b}$ \\
\hline & Ethambutol \\
\hline & Imipenem or meropenem \\
\hline & Para-aminosalicylic acid \\
\hline & Prothionamide \\
\hline & Pyrazinamide \\
\hline $\mathrm{C} 2$ & Delamanid ${ }^{c}$ \\
\hline
\end{tabular}

Modified from Korean guidelines for tuberculosis, 4th ed. [37].

${ }^{\text {a) }}$ The order of drug in group $\mathrm{C} 1$ does not mean the ranking of drug selection. ${ }^{b}$ Amikacin is preferred over streptomycin. Kanamycin can

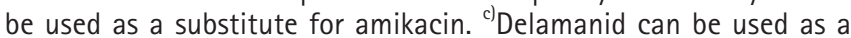
substitute for bedaquiline. 
drugs) at the initiation of the treatment. If regimens cannot be built based on the optimal regimen involving drugs from groups $\mathrm{A}$ and $\mathrm{B}$ due to drug resistance and toxicity, drugs from group $\mathrm{C}$ can be used. If the regimen cannot include all three agents from group A, initial treatment should be started with five agents, including all available agents in groups A and B. Injectable agents (amikacin or streptomycin), delamanid, pyrazinamide, or ethambutol can be chosen preferably. Susceptibility testing for fluoroquinolones should be performed prior to initiating MDR-TB treatment. Among the group A agents, fluoroquinolones have a high rate of resistance (up to 33\%) [57], and it is the only drug class for which rapid molecular tests are available. Resistance to fluoroquinolones was associated with poor outcomes (failure of treatment or relapse) in MDR-TB treatment [58]. Delamanid and second-line injectable drugs could be useful alternatives in fluoroquinolone-resistant MDR-TB. The possibility of treatment success in MDR-TB depends on patient factors (HIV infection, diabetes mellitus, low weight, large disease burden on chest radiography, genetic factors, and alcohol abuse), mycobacterial factors (resistance patterns, mycobacterial load), and optimal management (building of effective regimen and management of adverse effects and toxicities) [2,35]. American Thoracic Society, Centers for Disease Control and Prevention, European Respiratory Society, and Infectious Diseases Society of America (ATS/CDC/ERS/IDSA) published new guidelines for the treatment of drug-resistant-TB (including MDRTB and isoniazid-resistant TB) in 2019. The WHO and ATS/ $\mathrm{CDC} / \mathrm{ERS} / \mathrm{IDSA}$ guidelines were largely consistent, but there were some differences between two guidelines. ATS/CDC/ERS/ IDSA recommended larger number of drugs in building regimen and focused less on shorter regimen and injectable agents. ATS / $\mathrm{CDC} / \mathrm{ERS} / \mathrm{IDSA}$ guidelines recommended that the regimens should include at least five drugs at the initiation of the treatment and four drugs in the continuation phase [42]. These guidelines recommended six steps for building regimen: step 1, choose later generation of fluoroquinolone (levofloxacin or moxifloxacin); step 2 , choose both of the prioritized drugs (bedaquiline and linezolid); step 3, choose both of the two effective drugs (clofazimine and cycloserine); step 4, if a regimen cannot be built with five effective oral drugs, and the isolate is susceptible, use one of injectables (amikacin or streptomycin); step 5, if needed or if oral agents are preferred over injectable agents in step 4, injectables can be replaced by delamanid, pyrazinamide, or ethambutol; and step 6, if the options are limited, and a regimen of five effective drugs cannot be assembled, consider use of ethionamide/prothionamide, imipenem/meropenem plus clavulanate, para-aminosalicylic acid, or high-dose isoniazid [42].

\section{Duration of treatment}

The optimal duration of therapy for MDR-TB is unclear. The WHO recommends two types of standardized MDR-TB treatment regimens (longer and shorter regimens) [2]. They differ in drug combination as well as in duration. Treatment with the longer regimen is suggested for 18 to 20 months (at least 15 to 17 months after culture conversion), and oral regimens are preferred. The intensive phase, which lasts for 6 to 7 months and includes at least four drugs, is recommended until bedaquiline is stopped. The recommended duration of treatment may be modified depending on the culture conversion status and the patient's response to treatment [2]. The continuation phase of the treatment should include at least three drugs [2]. ATS/CDC/ERS/IDSA guidelines recommended the duration of intensive phase to be between 5 and 7 months after culture conversion [42].

The shorter regimen was originally based on the so-called Bangladesh regimen [59]. It was later tested in an international, randomized controlled trial (STREAM stage 1 trial) [60]. The recommended duration of this regimen is 9 to 11 months. The short regimen can be an alternative to the longer regimen in simple MDR-TB cases under specific conditions. This regimen includes an intensive phase lasting 4 to 6 months, which includes seven drugs (kanamycin, moxifloxacin, prothionamide, clofazimine, pyrazinamide, high-dose isoniazid, and ethambutol). It is followed by a 5-month course with moxifloxacin, clofazimine, pyrazinamide, and ethambutol. Exclusion criteria for the shorter regimen are (1) resistance to or suspected ineffectiveness of a medicine from the shorter regimen (except isoniazid resistance); (2) exposure to one or more second-line medicines from the shorter MDR-TB regimen for greater than 1 month; (3) intolerance to medicines from the shorter MDR-TB regimen or risk of toxicity (e.g., drug-drug interactions); (4) pregnancy; (5) disseminated, meningeal, or central nervous system TB; (6) any extrapulmonary disease in patients with HIV infection; and (7) unavailability of at least one medicine from the shorter MDR-TB regimen. ATS/CDC/ ERS/IDSA did not make a recommendation either for or against the standardized short-course regimen [42]. Korean guidelines also did not recommend shorter MDR-TB regimen because of the high incidence of resistance to quinolone, injectable agent, and pyrazinamide, and a lack of evidence on the effectiveness and safety of the shorter regimen when compared with the newly developed longer regimen [37].

\section{Conclusion}

MDR-TB remains a major concern in TB control. A rapid diagnosis of drug resistance and optimal treatment with effective and less toxic regimens is important in the management of MDR-TB. Re- 
cently, the WHO published updated guidelines regarding the programmatic management of MDR-TB, which focused on rapid diagnosis and effective treatment via advanced rapid molecular tests and oral regimens with new and repurposed anti-TB drugs. Using these current recommendations might be helpful in the management of MDR-TB. However, well-designed clinical trials and studies for further assessment of new agents and shorter regimens are needed.

\section{Acknowledgments}

\section{Conflicts of interest}

No potential conflict of interest relevant to this article was reported.

\section{Author contributions}

Conceptualization, Formal analysis, and Validation: JJG, CJH; Data curation, Methodology, Project administration, Visualization, Investigation, and Resources: JJG; Supervision: CJH; Writing-original draft: JJG, CJH; Writing-review \& editing: JJG, CJH.

\section{ORCID}

Jong Geol Jang, https://orcid.org/0000-0001-8040-5363 Jin Hong Chung, https://orcid.org/0000-0003-1829-3051

\section{References}

1. World Health Organization. Global health estimates 2016: deaths by cause, age, sex, by country and by region, 2000-2016 [Internet]. Geneva: World Health Organization; 2018 [cited 2020 Jul 14].https://www.who.int/healthinfo/global_burden_disease/estimates/en/.

2. World Health Organization. WHO consolidated guidelines on drug-resistant tuberculosis treatment [Internet]. Geneva: World Health Organization; 2019 [cited 2020 Jul 14]. https:// www.who.int/tb/publications/2019/consolidated-guidelines-drug-resistant-TB-treatment/en/.

3. World Health Organization. Global tuberculosis report 2015 [Internet]. 20th ed. Geneva: World Health Organization; 2015 [cited 2020 Jul 14]. https://apps.who.int/iris/handle/10665/ 191102.

4. World Health Organization. Global tuberculosis report 2019 [Internet]. Geneva: World Health Organization; 2019 [cited 2020 Jul 14].https://www.who.int/tb/publications/global_report/en/.

5. World Health Organization. Treatment guidelines for drug-resistant tuberculosis, 2016 update [Internet]. Geneva: World
Health Organization; 2016 [cited 2020 Jul 14]. https://www. who.int/publications/i/item/9789241549639.

6. David HL. Probability distribution of drug-resistant mutants in unselected populations of Mycobacterium tuberculosis. Appl Microbiol 1970;20:810-4.

7. Kim SJ. Drug-susceptibility testing in tuberculosis: methods and reliability of results. Eur Respir J 2005;25:564-9.

8. Zhang Y, Yew WW. Mechanisms of drug resistance in Mycobacterium tuberculosis: update 2015. Int J Tuberc Lung Dis 2015; 19:1276-89.

9. Kendall EA, Fofana MO, Dowdy DW. Burden of transmitted multidrug resistance in epidemics of tuberculosis: a transmission modelling analysis. Lancet Respir Med 2015;3:963-72.

10. Canetti G, Froman S, Grosset J, Hauduroy P, Langerova M, Mahler HT, et al. Mycobacteria: laboratory methods for testing drug sensitivity and resistance. Bull World Health Organ 1963;29:565-78.

11. World Health Oraganization. Guidelines for surveillance of drug resistance in tuberculosis [Internet]. 4th ed. Geneva: World Health Oraganization; 2009 [cited 2020 Jul 14]. https://www.who.int/ $\mathrm{tb} /$ publications/surveillance_guidelines/en/.

12. CanettI G, Rist N, Grosset J. Measurement of sensitivity of the tuberculous bacillus to antibacillary drugs by the method of proportions. Methodology, resistance criteria, results and interpretation. Rev Tuberc Pneumol (Paris) 1963;27:217-72.

13. Schaberg T, Reichert B, Schülin T, Lode H, Mauch H. Rapid drug susceptibility testing of Mycobacterium tuberculosis using conventional solid media. Eur Respir J 1995;8:1688-93.

14. World Health Organization. Use of liquid TB culture and drug susceptibility testing (DST) in low and medium income setting. Summary report of the Expert Group Meeting on the use of liquid culture media [Internet]. Geneva: World Health Organization; 2007 [cited 2018 Dec 18]. https://www.who.int/tb/laboratory/use_of_liquid_tb_culture_summary_report.pdf?ua $=1$.

15. Koh WJ, Ko Y, Kim CK, Park KS, Lee NY. Rapid diagnosis of tuberculosis and multidrug resistance using a MGIT 960 system. Ann Lab Med 2012;32:264-9.

16. Coll F, McNerney R, Preston MD, Guerra-Assunção JA, Warry A, Hill-Cawthorne G, et al. Rapid determination of anti-tuberculosis drug resistance from whole-genome sequences. Genome Med 2015;7:51.

17. World Health Organization. Molecular line probe assays for rapid screening of patients at risk of multidrug-resistant tuberculosis (MDR-TB) [Internet]. Geneva: World Health Organization; 2008 [cited 2020 Jul 14]. https://www.who.int/tb/laboratory/ line_probe_assays/en/.

18. World Health Organization. The use of molecular line probe as- 
says for the detection of resistance to isoniazid and rifampicin [Internet]. Geneva: World Health Organization; 2016 [cited 2020 Jul 14]. https://www.who.int/tb/publications/molecular-test-resistance/en/.

19. World Health Organization. WHO consolidated guidelines on tuberculosis. Module 3: diagnosis-rapid diagnostics for tuberculosis detection [Internet]. Geneva: World Health Organization; 2020 [cited 2020 Jul 14]. https://www.who.int/publications/i/item/who-consolidated-guidelines-on-tuberculosis-module-3-diagnosis---rapid-diagnostics-for-tuberculosis-detection.

20. Hillemann D, Rüsch-Gerdes S, Richter E. Evaluation of the GenoType MTBDRplus assay for rifampin and isoniazid susceptibility testing of Mycobacterium tuberculosis strains and clinical specimens. J Clin Microbiol 2007;45:2635-40.

21. Hain Lifescience GmbH. GenoType MTBDRplus, ver. 2.0. Instructions for use (IFU-304A-06) [Internet]. Nehren: Hain Lifescience GmbH; 2008 [cited 2020 Jul 14]. https://www. hain-lifescience.de/include_datei/kundenmodule/packungsbeilage $/$ download.php?id $=936$.

22. Ling DI, Zwerling AA, Pai M. GenoType MTBDR assays for the diagnosis of multidrug-resistant tuberculosis: a meta-analysis. Eur Respir J 2008;32:1165-74.

23. Theron G, Peter J, Richardson M, Barnard M, Donegan S, Warren R, et al. The diagnostic accuracy of the GenoType $\left({ }^{\circledR}\right)$ MTBDRsl assay for the detection of resistance to second-line anti-tuberculosis drugs. Cochrane Database Syst Rev 2014; (10):CD010705.

24. World Health Organization. Molecular assays intended as initial tests for the diagnosis of pulmonary and extrapulmonary $\mathrm{TB}$ and rifampicin resistance in adults and children: rapid communication [Internet]. Geneva: World Health Organization; 2020 [cited 2020 Jun 5]. https://apps.who.int/iris/handle/10665/ 330395.

25. El-Hajj HH, Marras SA, Tyagi S, Kramer FR, Alland D. Detection of rifampin resistance in Mycobacterium tuberculosis in a single tube with molecular beacons. J Clin Microbiol 2001; 39:4131-7.

26. Denkinger CM, Schumacher SG, Boehme CC, Dendukuri N, Pai M, Steingart KR. Xpert MTB/RIF assay for the diagnosis of extrapulmonary tuberculosis: a systematic review and meta-analysis. Eur Respir J 2014;44:435-46.

27. Dorman SE, Schumacher SG, Alland D, Nabeta P, Armstrong DT, King B, et al. Xpert MTB/RIF Ultra for detection of Mycobacterium tuberculosis and rifampicin resistance: a prospective multicentre diagnostic accuracy study. Lancet Infect Dis 2018;18:76-84.
28. Feliciano CS, Namburete EI, Rodrigues Plaça J, Peronni K, Dippenaar A, Warren RM, et al. Accuracy of whole genome sequencing versus phenotypic (MGIT) and commercial molecular tests for detection of drug-resistant Mycobacterium tuberculosis isolated from patients in Brazil and Mozambique. Tuberculosis (Edinb) 2018;110:59-67.

29. Witney AA, Cosgrove CA, Arnold A, Hinds J, Stoker NG, Butcher PD. Clinical use of whole genome sequencing for Mycobacterium tuberculosis. BMC Med 2016;14:46.

30. CRyPTIC Consortium and the 100,000 Genomes Project; Allix-Béguec C, Arandjelovic I, Bi L, Beckert P, Bonnet M, et al. Prediction of susceptibility to first-line tuberculosis drugs by DNA sequencing. N EnglJ Med 2018;379:1403-15.

31. Katale BZ, Mbelele PM, Lema NA, Campino S, Mshana SE, Rweyemamu MM, et al. Whole genome sequencing of Mycobacterium tuberculosis isolates and clinical outcomes of patients treated for multidrug-resistant tuberculosis in Tanzania. BMC Genomics 2020;21:174.

32. World Health Organization. The use of next-generation sequencing technologies for the detection of mutations associated with drug resistance in Mycobacterium tuberculosis complex: technical guide [Internet]. Geneva: World Health Organization; 2018 [cited 2020 Jul 14]. https://apps.who.int/iris/handle/10665/274443.

33. World Health Organization. Guidelines for the programmatic management of drug-resistant tuberculosis-2011 update [Internet]. Geneva: World Health Organization; 2011 [cited 2020 Jul 14]. https://apps.who.int/iris/bitstream/handle/10665/ 44597/9789241501583_eng.pdf?sequence $=1$.

34. World Health Organization. Rapid communication: key changes to treatment of multidrug- and rifampicin-resistant tuberculosis (MDR/RR-TB) [Internet]. Geneva: World Health Organization; 2011 [cited 2020 Jul 14]. https://www.who.int/tb/ publications/2018/rapid_communications_MDR/en/.

35. Collaborative Group for the Meta-Analysis of Individual Patient Data in MDR-TB treatment-2017; Ahmad N, Ahuja SD, Akkerman OW, Alffenaar JC, Anderson LF, et al. Treatment correlates of successful outcomes in pulmonary multidrug-resistant tuberculosis: an individual patient data meta-analysis. Lancet 2018;392:821-34.

36. Bastos ML, Lan Z, Menzies D. An updated systematic review and meta-analysis for treatment of multidrug-resistant tuberculosis. Eur Respir J 2017;49:1600803.

37. Joint Committee for the Revision of Korean Guidelines for Tuberculosis; Korean Centers for Disease Control and Prevention. Korean guidelines for tuberculosis. 4th ed. Cheongju: Korea Centers for Disease Control and Prevention; 2020. 
38. Moadebi S, Harder CK, Fitzgerald MJ, Elwood KR, Marra F. Fluoroquinolones for the treatment of pulmonary tuberculosis. Drugs 2007;67:2077-99.

39. Sirgel FA, Warren RM, Streicher EM, Victor TC, van Helden PD, Böttger EC. gyrA mutations and phenotypic susceptibility levels to ofloxacin and moxifloxacin in clinical isolates of Mycobacterium tuberculosis. J Antimicrob Chemother 2012;67: 1088-93.

40. Migliori GB, Langendam MW, D’Ambrosio L, Centis R, Blasi F, Huitric E, et al. Protecting the tuberculosis drug pipeline: stating the case for the rational use of fluoroquinolones. Eur Respir J 2012;40:814-22.

41. Kang YA, Shim TS, Koh WJ, Lee SH, Lee CH, Choi JC, et al. Choice between levofloxacin and moxifloxacin and multidrug-resistant tuberculosis treatment outcomes. Ann Am Tho$\operatorname{rac}$ Soc 2016;13:364-70.

42. Nahid P, Mase SR, Migliori GB, Sotgiu G, Bothamley GH, Brozek JL, et al. Treatment of drug-resistant tuberculosis. An official ATS/CDC/ERS/IDSA clinical practice guideline. Am J Respir Crit Care Med 2019;200:e93-142.

43. Livermore DM. Linezolid in vitro: mechanism and antibacterial spectrum. J Antimicrob Chemother 2013;51(Suppl 2):ii9-16.

44. Lan Z, Ahmad N, Baghaei P, Barkane L, Benedetti A, Brode SK, et al. Drug-associated adverse events in the treatment of multidrug-resistant tuberculosis: an individual patient data meta-analysis. Lancet Respir Med 2020;8:383-94.

45. Koh WJ, Kang YR, Jeon K, Kwon OJ, Lyu J, Kim WS, et al. Daily $300 \mathrm{mg}$ dose of linezolid for multidrug-resistant and extensively drug-resistant tuberculosis: updated analysis of 51 patients. J Antimicrob Chemother 2012;67:1503-7.

46. Sotgiu G, Centis R, D’Ambrosio L, Alffenaar JW, Anger HA, Caminero JA, et al. Efficacy, safety and tolerability of linezolid containing regimens in treating MDR-TB and XDR-TB: systematic review and meta-analysis. Eur Respir J 2012;40:1430 42.

47. Koul A, Dendouga N, Vergauwen K, Molenberghs B, Vranckx L, Willebrords R, et al. Diarylquinolines target subunit c of mycobacterial ATP synthase. Nat Chem Biol 2007;3:323-4.

48. Rouan MC, Lounis N, Gevers T, Dillen L, Gilissen R, Raoof A, et al. Pharmacokinetics and pharmacodynamics of TMC207 and its N-desmethyl metabolite in a murine model of tuberculosis. Antimicrob Agents Chemother 2012;56:1444-51.

49. Diacon AH, Pym A, Grobusch MP, de los Rios JM, Gotuzzo E, Vasilyeva I, et al. Multidrug-resistant tuberculosis and culture conversion with bedaquiline. N Engl J Med 2014;371:723-32.

50. Diacon AH, Pym A, Grobusch M, Patientia R, Rustomjee R, Page-Shipp L, et al. The diarylquinoline TMC207 for multidrug-resistant tuberculosis. N Engl J Med 2009;360:2397-405.

51. Centers for Disease Control and Prevention. Provisional CDC guidelines for the use and safety monitoring of bedaquiline fumarate (Sirturo) for the treatment of multidrug-resistant tuberculosis. MMWR Recomm Rep 2013;62(RR-09):1-12.

52. Matsumoto M, Hashizume H, Tomishige T, Kawasaki M, Tsubouchi H, Sasaki H, et al. OPC-67683, a nitro-dihydro-imidazooxazole derivative with promising action against tuberculosis in vitro and in mice. PLoS Med 2006;3:e466.

53. Barry PJ, O'Connor TM. Novel agents in the management of Mycobacterium tuberculosis disease. Curr Med Chem 2007; $14: 2000-8$.

54. Kim CT, Kim TO, Shin HJ, Ko YC, Choe YH, Kim HR, et al. Bedaquiline and delamanid for the treatment of multidrug-resistant tuberculosis: a multicentre cohort study in Korea. Eur Respir J 2018;51:1702467.

55. MokJ, Kang H, Koh WJ, Jhun BW, Yim JJ, Kwak N, et al. Final treatment outcomes of delamanid-containing regimens in patients with MDR-/XDR-TB in South Korea. Eur Respir J 2019; 54:1900811.

56. Ferlazzo G, Mohr E, Laxmeshwar C, Hewison C, Hughes J, Jonckheere S, et al. Early safety and efficacy of the combination of bedaquiline and delamanid for the treatment of patients with drug-resistant tuberculosis in Armenia, India, and South Africa: a retrospective cohort study. Lancet Infect Dis 2018;18:53644.

57. Lange C, Duarte R, Fréchet-Jachym M, Guenther G, Guglielmetti L, Olaru ID, et al. Limited benefit of the new shorter multidrug-resistant tuberculosis regimen in Europe. Am J Respir Crit Care Med 2016;194:1029-31.

58. Cegielski JP, Kurbatova E, van der Walt M, Brand J, Ershova J, Tupasi T, et al. Multidrug-resistant tuberculosis treatment outcomes in relation to treatment and initial versus acquired second-line drug resistance. Clin Infect Dis 2016;62:418-30.

59. Van Deun A, Maug AK, Salim MA, Das PK, Sarker MR, Daru $\mathrm{P}$, et al. Short, highly effective, and inexpensive standardized treatment of multidrug-resistant tuberculosis. Am J Respir Crit Care Med 2010;182:684-92.

60. Nunn AJ, Phillips PP, Meredith SK, Chiang CY, Conradie F, Dalai $\mathrm{D}$, et al. A trial of a shorter regimen for rifampin-resistant tuberculosis. N Engl J Med 2019;380:1201-13. 